\title{
Hiperglucemia intrahospitalaria durante la COVID-19 en pacientes sin diagnóstico previo de diabetes: reporte de tres casos
}

\section{Hospital hyperglycemia during COVID-19 in patients without a previous diagnosis of diabetes: A report of three cases}

\author{
Gustavo Inzunza-Cervantes ${ }^{1 *}$, Rocío M. López-López ${ }^{1}$, José M. Ornelas-Aguirre², Gabriela V. Flores-Montes ${ }^{1}$, \\ marco A. Ponce-Peraza ${ }^{1}$ y Alibe N. Peña-Valenzuela ${ }^{3}$
}

1Servicio de Medicina Interna, Hospital de Especialidades N. ${ }^{\circ}$ 2, Centro Médico Nacional del Noroeste, Instituto Mexicano del Seguro Social; ${ }^{2}$ Departamento de Ciencias de la Salud, Universidad de Sonora; ${ }^{3}$ Unidad Médico Familiar N. ${ }^{1}$, Instituto Mexicano del Seguro Social. Ciudad Obregón, Sonora, México

\section{RESUMEN}

\begin{abstract}
Introducción: Durante la pandemia por enfermedad por coronavirus 2019 (COVID-19) se ha reportado un incremento de casos de hiperglucemia hospitalaria en pacientes con y sin diabetes. Como factores desencadenantes se ha sugerido el uso de glucocorticoides, el estado hiperinflamatorio, el daño a las células beta del páncreas, la ansiedad, el antecedente de diabetes mellitus y la disfunción del tejido adiposo. Se describen tres casos de pacientes que cursaron con hiperglucemia asociada a la COVID-19. Métodos: Mediante la presentación de casos clínicos y revisión de la literatura, se investigaron elementos claves que mejoren el entendimiento, diagnóstico y tratamiento de la hiperglucemia hospitalaria en pacientes con COVID-19 sin diagnóstico previo de diabetes mellitus. Conclusiones: El incremento de casos de hiperglucemia intrahospitalaria en pacientes con COVID-19 requiere de un diagnóstico y tratamiento oportuno, dadas las implicaciones negativas de esta entidad; para su abordaje se aconseja la medición de glucemia sérica, hemoglobina glucosilada y en casos selectos enzimas pancreáticas. Su tratamiento se basa en el empleo de insulina en diferentes esquemas de tratamiento y vigilancia estrecha del paciente. Previo a su egreso se debe evaluar la terapia hipoglucemiante más adecuada para su manejo ambulatorio.
\end{abstract}

Palabras clave: Hiperglucemia intrahospitalaria. COVID-19. Diabetes mellitus. SARS-CoV-2.

\begin{abstract}
Introduction: During the coronavirus disease 2019 (COVID-19) pandemic, an increase in cases of hospital hyperglycemia has been reported in patients with and without diabetes. The use of glucocorticoids, the hyperinflammatory state, damage to the beta cells of the pancreas, anxiety, a history of diabetes mellitus and adipose tissue dysfunction have been suggested as causes. Three cases of patients with hyperglycemia associated with COVID-19 are described. Methods: By presenting clinical cases and reviewing the literature, were investigated key elements that improve the understanding, diagnosis, and treatment of hospital hyperglycemia in patients with COVID-19 without a previous diagnosis of diabetes mellitus. Conclusions: The increase in cases of intra-hospital hyperglycemia in patients with COVID-19 requires opportune diagnosis and treatment, given the negative implications of this entity, for its approach it is advisable to measure serum glycemia, glycated hemoglobin and in selected cases pancreatic enzymes. Its treatment is based on the use of insulin in different treatment schemes and near monitoring of the patient. Before discharge, the most appropriate hypoglycemic therapy for outpatient management should be evaluated.
\end{abstract}

Key words: In-hospital hyperglycemia. COVID-19. Diabetes mellitus. SARS-CoV-2.
Correspondencia:

*Gustavo Inzunza-Cervantes

E-mail: gusinzunza@live.com.mx
Fecha de recepción: 02-10-2020

Fecha de aceptación: 05-02-2021

DOI: 10.24875/RME.20000095
Disponible en internet: 03-03-2021

Rev Mex Endocrinol Metab Nutr. 2021;8:134-42

2462-4144 / @ 2021 Sociedad Mexicana de Nutricion y Endocrinologia, AC. Publicado por Permanyer. Este es un artículo open access bajo la licencia CC BY-NC-ND (http://creativecommons.org/licenses/by-nc-nd/4.0/). 


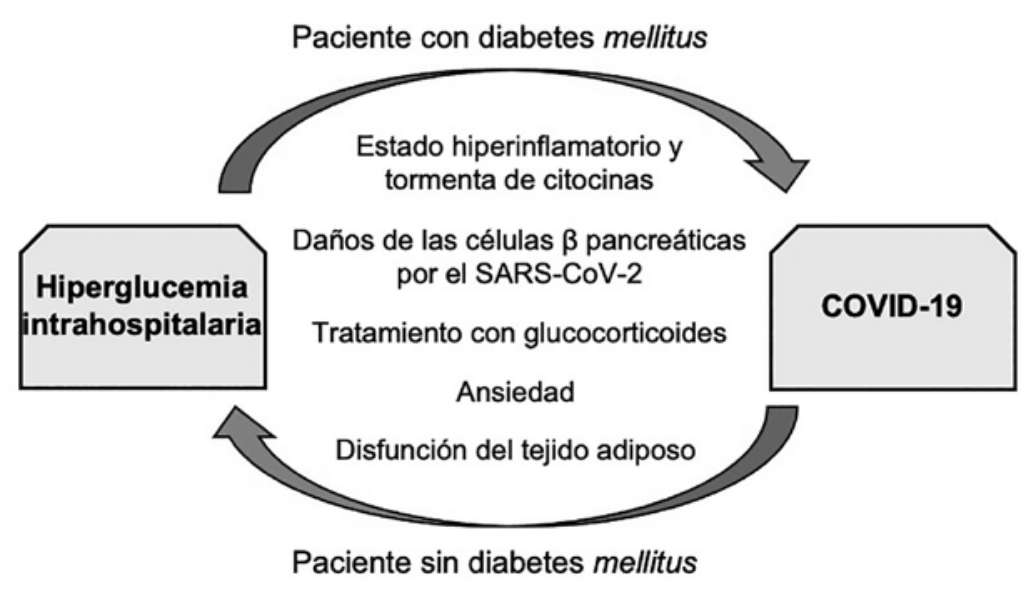

Figura 1. Factores relacionados con el desarrollo de hiperglucemia en pacientes diabéticos y no diabéticos hospitalizados por COVID-19. COVID-19: enfermedad por coronavirus 2019; SARS-CoV-2: coronavirus 2 del síndrome respiratorio agudo grave.

\section{INTRODUCCIÓN}

Durante la pandemia por COVID-19 se ha reportado un incremento de hiperglucemia hospitalaria en pacientes con diabetes y sin diabetes ${ }^{1,2}$, asociándose a un estado hiperinflamatorio ${ }^{3}$, uso de esteroides, daño de las células beta en el páncreas por el coronavirus 2 del síndrome respiratorio agudo grave (SARS-CoV-2), ansiedad, diabetes mellitus y disfunción del tejido adiposo ${ }^{4,5}$. Se considera una complicación común, grave y de alto costo en los pacientes hospitalizados por COVID-19 que incrementa la estancia hospitalaria, mortalidad y ventilación mecánica (odds ratio [OR]: 5.47; intervalo de confianza del 95\% [IC 95\%]:) ${ }^{6-8}$ (Fig. 1).

Estudios previos sugieren que al mejorar el control glucémico se logran tasas más bajas de complicaciones. Por tal motivo, se ha sugerido la implementación de programas de atención que incluyan terapia hipoglucemiante y apoyo nutricional adecuado para el enfermo por COVID-19 hospitalizado ${ }^{9,10}$.

\section{MÉTODOS}

Se realizó un estudio analizando los registros clínicos de los enfermos y describiendo a aquellos que cursaron con hiperglucemia durante su hospitalización por COVID-19. Ninguno de los casos que se presentan tenían antecedente de diabetes mellitus; todos fueron atendidos en el área COVID-19 del Servicio de Medicina Interna del Hospital de Especialidades N. 2 en el Centro Médico Nacional del Noroeste del Instituto Mexicano del Seguro Social en Ciudad Obregón, Sonora, México. En una ficha predefinida se registraron datos clínicos, paraclínicos, tratamientos complementarios y evolución de los pacientes. La literatura científica analizada se obtuvo con la finalidad de explorar elementos claves que mejoren el entendimiento, diagnóstico y tratamiento de la hiperglucemia hospitalaria en pacientes con COVID-19 sin diagnóstico previo de diabetes mellitus, realizando una búsqueda con los términos: hiperglucemia intrahospitalaria, COVID-19, diabetes mellitus y disglucemia en COVID-19 en las bases de datos de Medline (PubMed), Scientific Electronic Library Online (SciELO), Índice de Revistas Médicas Latinoamericanas (IMBIOMED), New England Journal of Medicine, The Lancet, Nature y Science, inicialmente publicadas durante el periodo 2019 al 2020 referente a la COVID-19, complementando con literatura sobre el tema publicado entre 2010 y 2020 en los idiomas inglés y español. Cada uno de los artículos se revisó de forma minuciosa en relación con la epidemiología, fisiopatología, manifestaciones clínicas, diagnóstico y tratamiento de la hiperglucemia en la COVID-19, seleccionando aquello que por su contenido contribuía al objetivo de la investigación. 


\section{REPORTE DE LOS CASOS CLÍNICOS}

\section{Caso 1}

Paciente de sexo masculino de 61 años de edad diagnosticado con neumonía atípica por SARS-CoV-2, fumador crónico, sin antecedentes de enfermedades cronicodegenerativas, que acudió por un cuadro de mal estado general, hiporexia, cefalea, sensación térmica elevada y disnea de seis días; desde su ingreso requirió oxígeno suplementario, inicialmente través de puntas nasales a dosis de $3 \mathrm{l} / \mathrm{min}$ y posteriormente con mascarilla reservorio para mantener saturaciones de oxígeno > 88\%; en sus laboratorios iniciales, tomados a su ingreso a urgencias destaca la presencia de hiperglucemia de $352 \mathrm{mg} / \mathrm{dL}$, registros de glucemias capilares prepandiales por glucometría $>200 \mathrm{mg} / \mathrm{dL}$ con posterior glucemia en ayuno de $322 \mathrm{mg} / \mathrm{dL}$, decidiéndose manejo con insulina esquema basal-bolo ajustado a tasa de filtrado glomerular, calculándose a $0.3 \mathrm{UI} / \mathrm{kg}$ de peso (glargina a dosis de $18 \mathrm{UI}$ e insulina lispro a dosis de $6 \mathrm{UI}$ cada $8 \mathrm{~h}$ ). El paciente persistió fuera de metas, requiriendo incremento del $20 \%$ de la dosis total de insulina, normalizándose glucemias séricas en ayuno (176 mg/dL) y glucosas capilares por glucometría al 4. ${ }^{\circ}$ día de iniciado esquema de insulina. Ante estos hallazgos se inició abordaje de estudio, reportándose hemoglobina glucosilada (HbA1c) del $13.6 \%$. Se diagnosticó hiperglucemia secundaria a diabetes mellitus no diagnosticada, a pesar de que sus glucemias séricas y capilares prepandiales se normalizaron. En el 5. ${ }^{\circ}$ día de estancia presentó deterioro respiratorio que requirió de apoyo ventilatorio, con posterior desenlace fatal (Tabla 1).

\section{Caso 2}

Mujer de 67 años de edad ingresada a área de hospitalización COVID-19 por neumonía atípica por SARSCoV-2. Dentro de sus antecedentes de interés destacan: exposición a inhalación de humo de leña, artritis reumatoide de 20 años de evolución, con mala adherencia terapéutica, ingiriendo de manera ocasional ante presencia de dolor diclofenaco tabletas $100 \mathrm{mg}$. Se remite a hospitalización por presentar mal estado general, tos no productiva, cefalea y disnea de 10 días de evolución; durante los dos primeros días necesitó oxígeno con puntas nasales a dosis de $5 \mathrm{l} / \mathrm{min}$ para mantener saturaciones $>88 \%$; dentro de sus paraclínicos iniciales se reportó una glucosa sérica al azar de $410 \mathrm{mg} / \mathrm{dL}$, con posterior glucosa sérica en ayuno de $311 \mathrm{mg} / \mathrm{dL}$ y glucosas capilares prepandiales medidas por glucometría $>200 \mathrm{mg} / \mathrm{dL}$, requiriendo esquema de insulina en esquema bolo-basal a dosis de $0.5 \mathrm{UI} / \mathrm{kg}$ de peso (glargina $12 \mathrm{UI}$, lispro $4 \mathrm{UI}$ cada $8 \mathrm{~h}$ ). Como parte del abordaje diagnóstico de hiperglucemia hospitalaria en paciente con COVID-19 se solicitó HbA1c, reportándose del $10.1 \%$, con una amilasa sérica de $15 \mathrm{UI} / \mathrm{l}$ y lipasa de $24 \mathrm{UI} / \mathrm{l}$. Se confirmó que la hiperglucemia era secundaria a diabetes mellitus no diagnosticada. La paciente presentó buena evolución del cuadro respiratorio, normalizándose glucemias y egresándose del hospital a los siete días, con mismo esquema de insulina, estando pendiente su evaluación en la consulta externa de medicina interna para ajustes a 1 mes después de su alta (Tabla 1).

\section{Caso 3}

Paciente de sexo femenino de 54 años de edad diagnosticada con neumonía atípica por SARS-CoV-2, que presenta como antecedente médico de importancia asma bronquial de 17 años de evolución bajo tratamiento con salbutamol en aerosol a libre demanda. Acudió a atención médica por presentar sensación térmica elevada, mal estado general, cefalea y disnea de 8 días de evolución tratadas previamente de manera ambulatoria con 4 dosis de ceftriaxona de $1 \mathrm{~g}$ i.v. c/24 h, enoxaparina a dosis de 60 UI subcutáneas cada 24 horas y dexametasona a dosis de $6 \mathrm{mg}$ día i.v. por 4 días. Los primeros días de estancia hospitalaria requirió oxígeno suplementario inicialmente con mascarilla y reservorio a dosis de $14 \mathrm{l} / \mathrm{min}$ y posterior con puntas nasales. Los estudios de laboratorio iniciales tomados en urgencias evidenciaban leucocitosis a expensas de linfocitosis e hiperglucemia de $190 \mathrm{mg} / \mathrm{dL}$, posterior glucemia sérica en ayuno en $180 \mathrm{mg} / \mathrm{dL}$, al segundo día de estancia se normalizan glucemias séricas en ayuno $(142 \mathrm{mg} / \mathrm{dL})$ y glucosas capilares medidas por glucometría, la HbA1c se reportó en $6.4 \%$, con amilasa de $47 \mathrm{UI} / \mathrm{l}$ y una lipasa sérica de $26 \mathrm{UI} / \mathrm{l}$. En esta paciente por definición 
Tabla 1. Información paraclínica y tratamiento de los casos clínicos estudiados

\begin{tabular}{|c|c|c|c|}
\hline & Caso 1 & Caso 2 & Caso 3 \\
\hline $\begin{array}{l}\text { Glucemias al azar tomadas } \\
\text { a su ingreso urgencias }\end{array}$ & $352 \mathrm{mg} / \mathrm{dL}$ & $410 \mathrm{mg} / \mathrm{dL}$ & $190 \mathrm{mg} / \mathrm{dL}$ \\
\hline $\begin{array}{l}\text { Glucemias séricas en } \\
\text { ayuno }\end{array}$ & $\begin{array}{l}322 \mathrm{mg} / \mathrm{dL} \\
272 \mathrm{mg} / \mathrm{dL} \\
176 \mathrm{mg} / \mathrm{dL}\end{array}$ & $\begin{array}{l}311 \mathrm{mg} / \mathrm{dL} \\
168 \mathrm{mg} / \mathrm{dL}\end{array}$ & $\begin{array}{l}180 \mathrm{mg} / \mathrm{dL} \\
142 \mathrm{mg} / \mathrm{dL}\end{array}$ \\
\hline $\mathrm{HbA1c}$ & $13.6 \%$ & $10.1 \%$ & $6.4 \%$ \\
\hline Enzimas pancreáticas & $\begin{array}{l}\text { Amilasa } 29 \mathrm{UI} / \mathrm{L} \\
\text { Lipasa } 22 \mathrm{UI} / \mathrm{L}\end{array}$ & $\begin{array}{l}\text { Amilasa } 15 \mathrm{UI} / \mathrm{L} \\
\text { Lipasa } 24 \mathrm{UI} / \mathrm{L}\end{array}$ & $\begin{array}{l}\text { Amilasa } 47 \mathrm{UI} / \mathrm{L} \\
\text { Lipasa } 26 \mathrm{UI} / \mathrm{L}\end{array}$ \\
\hline Laboratorios adicionales & PCR para SARS-CoV-2 positiva & PCR para SARS-CoV-2 positiva & PCR para SARS-CoV-2 positiva \\
\hline Laboratorios adicionales & $\begin{array}{l}\text { Urea } 89 \mathrm{mg} / \mathrm{dL}, \text { BUN } 42 \mathrm{mg} / \mathrm{dL} \text {, } \\
\mathrm{Cr} 1.47 \mathrm{mg} / \mathrm{dL}, \mathrm{Hb} 12.3 \mathrm{~g} / \mathrm{dL}, \\
\text { leucocitos 10,300 } 10^{3 /} \mu \mathrm{L} \text {, neutrofilos } \\
8,80010^{3 /} \mu \mathrm{L} \text {, linfocitos } 7,80010^{3 /} \mu \mathrm{L} \text {, } \\
\text { plaquetas } 233,00010^{3 /} \mu \mathrm{L}, \mathrm{AST} \\
88 \mathrm{UI} / \mathrm{L}, \mathrm{ALT} 69 \mathrm{UI} / \mathrm{L} \text {, albumina } \\
2.94 \mathrm{~g} / \mathrm{dL} \text { DHL } 351 \mathrm{UI} / \mathrm{L}\end{array}$ & $\begin{array}{l}\text { Urea } 21 \mathrm{mg} / \mathrm{dL}, \text { BUN10 mg/dL, } \\
\text { Cr } 0.71 \mathrm{mg} / \mathrm{dL}, \mathrm{Hb} 11.2 \mathrm{~g} / \mathrm{dL}, \\
\text { leucocitos 8,700 } 10^{3 /} \mu \mathrm{L}, \\
\text { neutrofilos } 7,00010^{3 /} \mu \mathrm{L}, \\
\text { plaquetas } 501,00010^{3 /} \mu \mathrm{L}, \\
\mathrm{K} 4 \mathrm{mmol} / \mathrm{L}, \mathrm{Na} 135 \mathrm{mmol} / \mathrm{L}, \\
\text { AST11 UI/L, albumina } 2.8 \mathrm{~g} / \mathrm{dL},\end{array}$ & 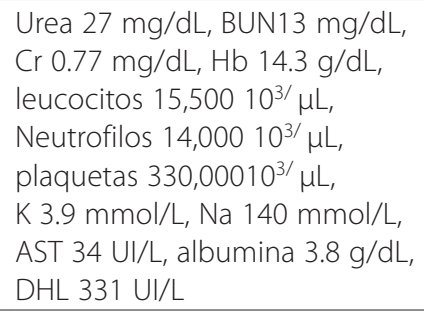 \\
\hline $\begin{array}{l}\text { Tasa de filtrado glomerular } \\
\text { por MDR/CKD-EPI }\end{array}$ & $50.8 \mathrm{~mL} / \mathrm{min} / 1.73 \mathrm{~m}^{2}$ & $111.9 \mathrm{~mL} / \mathrm{min} / 1.73 \mathrm{~m}^{2}$ & $101.5 \mathrm{~L} / \mathrm{min} / 1.73 \mathrm{~m}^{2}$ \\
\hline Radiografía de Tórax & $\begin{array}{l}\text { Múltiples opacidades reticulares en } \\
\text { campos superiores y medios de } \\
\text { ambos pulmones }\end{array}$ & $\begin{array}{l}\text { Opacidades en vidrio esmerilado } \\
\text { bilaterales }\end{array}$ & $\begin{array}{l}\text { Opacidades en vidrio } \\
\text { esmerilado en base de pulmón } \\
\text { derecho }\end{array}$ \\
\hline $\begin{array}{l}\text { Tratamiento } \\
\text { Hipoglucemiante }\end{array}$ & $\begin{array}{l}\text { Insulina glargina } 18 \text { UI y lispro } \\
\text { 6-6-6 UI SC } \\
\text { Ajuste: insulina glargina } 20 \mathrm{UI} \text { y } \\
\text { lispro 7-7-7 UI SC }\end{array}$ & $\begin{array}{l}\text { Insulina glargina } 12 \text { UI SC y } \\
\text { lispro 4-4-4 UI SC }\end{array}$ & Apoyo nutricional \\
\hline Tratamiento integral & $\begin{array}{l}\text { Enoxaparina } 60 \text { UI SC c/12 h } \\
\text { Dexametasona } 6 \text { mg IV c/24 h } \\
10 \text { días } \\
\text { Paracetamol tab } 500 \text { mg c/8 h } \\
\text { Propofol infusion } 2.8 \mathrm{mg} / \mathrm{kg} / \mathrm{h} \\
\text { Dexmetomidina infusion } \\
0.7 \mu \mathrm{g} / \mathrm{kg} / \mathrm{h} \\
\text { Buprenorfina infusión } \\
0.5 \mu \mathrm{g} / \mathrm{kg} / \mathrm{h}\end{array}$ & $\begin{array}{l}\text { Enoxaparina } 60 \mathrm{UI} \text { SC c/24 h } \\
\text { Dexametasona } 6 \mathrm{mg} \mathrm{IV} \mathrm{c/24} \mathrm{h} \\
10 \text { días } \\
\text { Paracetamol tab } 500 \mathrm{mg} \mathrm{c/8} \mathrm{h} \\
\text { Benzonatato perlas } 100 \mathrm{mg} \\
1 \mathrm{c} / 8 \mathrm{~h}\end{array}$ & $\begin{array}{l}\text { Enoxaparina } 60 \text { UI SC c/24 h } \\
\text { Dexametasona } 6 \text { mg IV c/24 h } \\
10 \text { días } \\
\text { Paracetamol tab } 500 \text { mg c/8 h } \\
\text { Budesonide- formoterol aerosol } \\
160 / 4.5 \text { mcg dosificación c/12 h }\end{array}$ \\
\hline
\end{tabular}

HbA1c: hemoglobina glucosilada; Hb: hemoglobina; MDRD/CKD-EPI: Modification of Diet in Renal Disease/Chronic Kidney Disease Epidemiology Collaboration; PCR: reacción en cadena de la polimerasa; SARS-CoV-2: coronavirus 2 del síndrome respiratorio agudo grave; tab: tableta; BUN: nitrógeno ureico en sangre; Cr: creatinina; AST: aspartato aminotransferasa; ALT: alanina aminotransferasa; DHL: deshidrogenasa láctica; K: potasio; Na: sodio; IV: intravenosa; mg/dL: miligramos por decilitro; UI/L: unidades internacionales por litro; SC: subcutánea; mcg: microgramo; mL/min: mililitros/minuto.

teórica se confirmó hiperglucemia no relacionada con diabetes mellitus y probablemente secundaria al uso de glucocorticoides, sin embargo, debido a valores limítrofes se consideró como intolerancia a la glucosa, otorgándose apoyo nutricional-dietético. Presentó una buena evolución del cuadro respiratorio, egresándose con manejo ambulatorio nutricional, cambio de broncodilatadores a budesonida formoterol aerosol acorde a guías GINA (Global Initiative for
Asthma) y cita en un mes al servicio de medicina interna, estando vigente su revaloración (Tabla 1).

\section{DISCUSIÓN}

Se define hiperglucemia intrahospitalaria cuando el valor de glucosa en sangre es mayor a $140 \mathrm{mg} / \mathrm{dL}$ y 


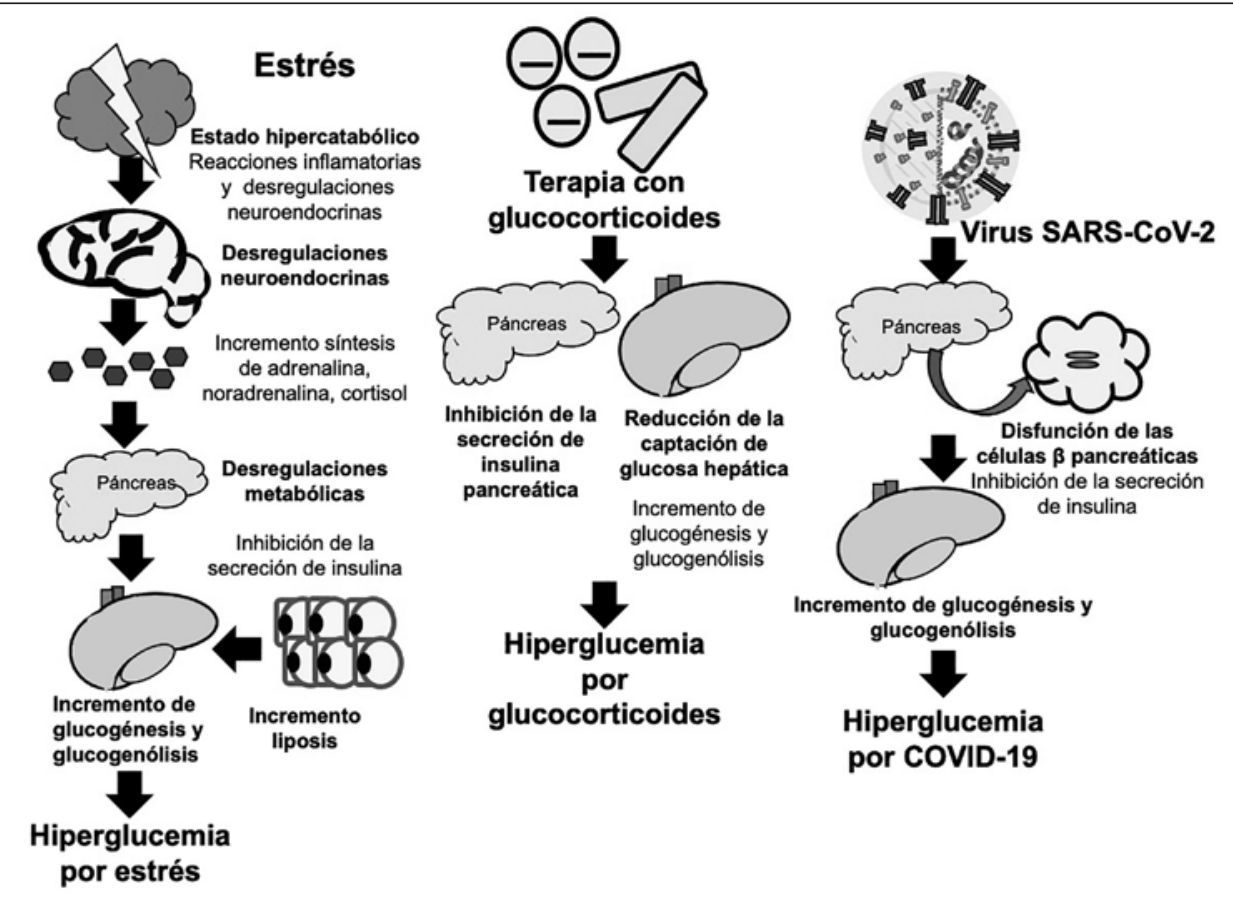

Figura 2. Fisiopatología asociada al origen postestrés, uso de glucocorticoides e infección por SARS-CoV-2. Se describe la fisiopatología de los principales factores relacionados con hiperglucemia intrahospitalaria no relacionada con diabetes: hiperglucemia por estrés, hiperglucemia por glucocorticoides e hiperglucemia por COVID-19. COVID-19: enfermedad por coronavirus 2019; SARS-CoV-2: coronavirus 2 del síndrome respiratorio agudo grave.

puede ocurrir en pacientes con y sin diabetes mellitus $^{11,12}$, reportándose por Bello-Chavolla, et al. una prevalencia en México de diabetes del 18\% en los pacientes hospitalizados por COVID-1913, concordante con el $21 \%$ reportado a nivel mundial ${ }^{14}$, y entre el 20 y el $40 \%$ desarrollan hiperglucemia. Esta cifra puede llegar hasta un $70 \%$ en los pacientes críticamente enfermos independientemente de la presencia de diabetes mellitus previamente conocida ${ }^{15}$.

La hiperglucemia puede estar asociada a diabetes mellitus (sea esta con diagnóstico previo o sin diagnóstico anterior conocido, pero que son portadores de la enfermedad), o no estar relacionadas con diabetes mellitus (como hiperglucemia por estrés) ${ }^{15,16}$, uso de glucocorticoides ${ }^{17-19} \mathrm{y}$, más recientemente, por COVID-1920-22 (Fig. 2).

Debido a esta reciente relación de hiperglucemia hospitalaria con COVID 19, solicitamos de manera intencionada estudios paraclínicos que permitieran documentar su presencia. El mecanismo fisiopatológico planteado mediante el cual el SARS-CoV-2 causa disfunción pancreática aguda se debe al tropismo de este virus sobre los receptores de la enzima convertidora de angiotensina pancreáticos, generando daño directo a los islotes de células de Langerhans, lo que reduce la secreción de insulina y causa hiperglucemia ${ }^{21,22}$.

Se ha sugerido que en los pacientes diabéticos la infección por SARS-CoV-2 se asocia a descontrol glucémico más allá de lo que contribuye la hiperglucemia por estrés, y a su vez la diabetes mellitus exacerba la gravedad de la COVID-19, debido a una mayor susceptibilidad de desarrollar tormenta de citocinas inflamatorias ${ }^{5,23}$.

En todo paciente con hiperglucemia intrahospitalaria se debe solicitar $\mathrm{HbA} 1 \mathrm{c}^{11}$, en pacientes que no son diabéticos (como los casos 1 y 2 ) un valor mayor al $6.5 \%$ sugiere la presencia de diabetes no diagnosticada previa a la hospitalización ${ }^{24,25}$. Debe tenerse en cuenta que el valor puede alterarse por la presencia de anemia, transfusiones sanguíneas, enfermedad renal o hepática grave ${ }^{26}$. La presencia de niveles elevados de amilasa, lipasa y cambios focales en el páncreas en pacientes que cursan con infección por 


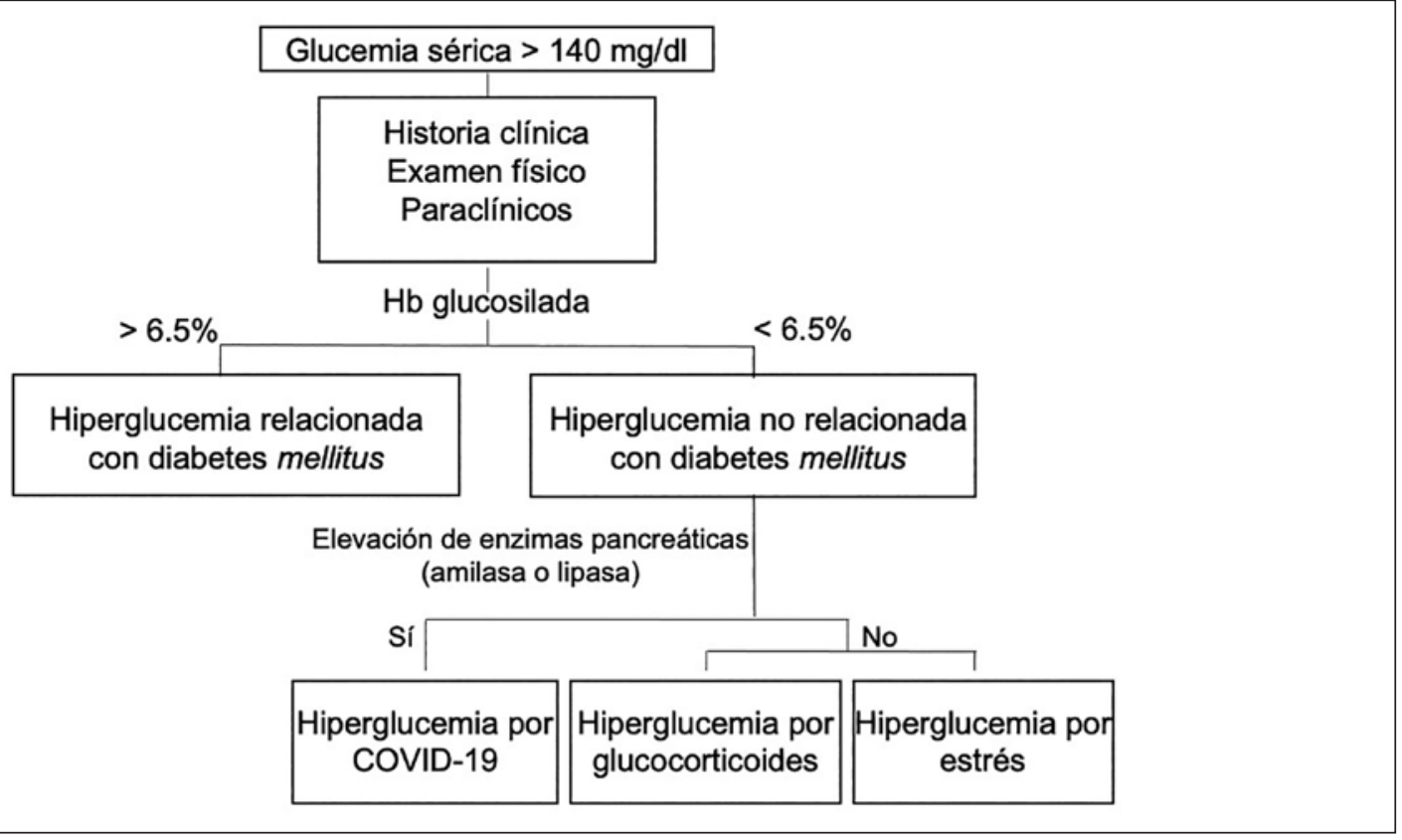

Figura 3. Abordaje diagnóstico en la hiperglucemia intrahospitalaria en paciente sin diagnóstico previo de diabetes con COVID-19. Hb: hemoglobina; COVID-19: enfermedad por coronavirus 2019.

SARS-CoV-2 han sido reportados en afectación pancreática por COVID-19, por lo que su incremento en pacientes con hiperglucemia sugiere disfunción pancreática asociada a infección por SARS-CoV-2, situación ausente en los casos presentados ${ }^{27,28}$ (Fig. 3).

Las metas en el control glucémico determinadas en los casos clínicos presentados partió de las recomendaciones internacionales que sugieren en pacientes hospitalizados no críticos niveles de glucosa en ayuno menores a $140 \mathrm{mg} / \mathrm{dL}$ y glucosa al azar menores a $180 \mathrm{mg} / \mathrm{dL}$; reportándose en pacientes críticos mayor beneficio con controles glucémicos más estrictos, de 110 a $140 \mathrm{mg} / \mathrm{dL}^{11,15}$.

Para el tratamiento de la hiperglucemia intrahospitalaria la insulina es el agente más apropiado en la mayoría de los casos; sin embargo, en el contexto de la infección aguda por SARS-CoV-2 existe poca información sobre los beneficios o riesgos de esta práctica, partiendo de las recomendaciones de su uso actual de estudios previos ${ }^{29,30}$. De manera general las diferentes guías internacionales recomiendan en pacientes críticos el uso de insulina en infusión intravenosa continua y en los pacientes hospitalizados no críticos, el uso de esquemas subcutáneos, siendo el esquema de insulina basalbolo (prandial) el de elección ${ }^{31-34}$. No se aconseja el uso exclusivo de insulina de escala móvil ${ }^{29}$. La terapia basal consta de una insulina de acción intermedia o prolongada (glargina, detemir, NPH [(protamina neutra de Hagedorn)]) para suprimir la liberación hepática de glucosa durante el ayuno y entre los alimentos, el régimen de bolo consiste en insulina de acción rápida (aspart, lispro, glulisina), que previene los picos posprandiales de glucosa y el régimen de corrección se otorga con insulina regular o análoga de acción rápida como dosis suplementaria para disminuir la glucosa que se encuentra superior a los rangos de control ${ }^{20,31}$.

Para inicio del esquema basal-bolo de insulina en los casos clínicos presentados se consideraron las siguientes recomendaciones: ${ }^{35}$ en el caso 1 debido a que la tasa de filtración glomerular fue menor a $60 \mathrm{ml} / \mathrm{min} / \mathrm{m}^{2}$ se calculó la dosis total de insulina a razón de $0.3 \mathrm{UI} / \mathrm{kg}$, otra consideración para realizar este ajuste es en pacientes mayores de 70 años. En los pacientes que no cumplan los criterios antes descritos (como los casos 2 y 3 ) se deberá de calcular a dosis de $0.4 \mathrm{Ul} / \mathrm{kg}$ si la glucemia al ingreso está entre 140 y $200 \mathrm{mg} / \mathrm{dL}$ o cercana a $0.5 \mathrm{Ul} / \mathrm{kg}$ en pacientes 


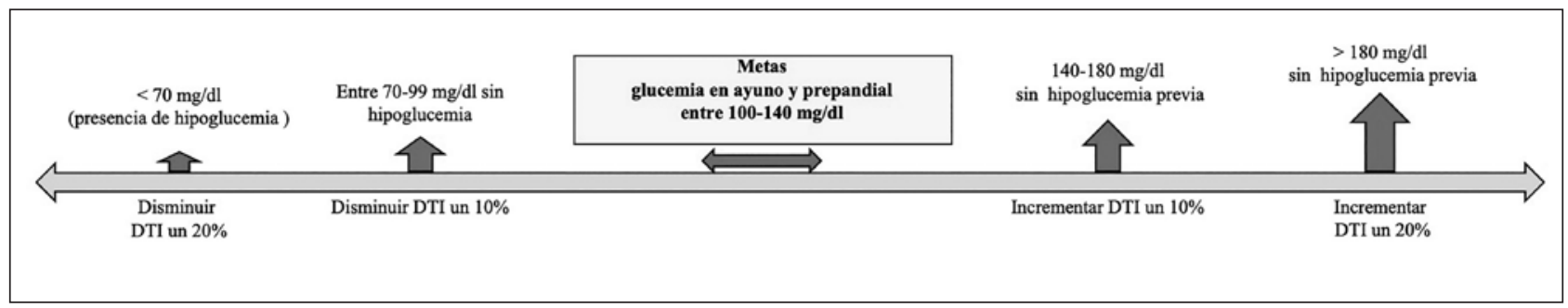

Figura 4. Monitorización y ajuste de insulina acorde a los resultados de glucemia en ayuno y prepandial. DTI: dosis total de insulina.

con glucemia al ingreso de entre 201 y $400 \mathrm{mg} / \mathrm{dL}$; la distribución de dosis total de insulina se calculó en un $50 \%$ de insulina basal y $50 \%$ de insulina preprandial, esta última dividida en tres partes iguales para cada una de las comidas principales. Deberá considerarse que los pacientes con diabetes que padecen COVID-19 pueden requerir una dosis alta de insulina para controlar la glucemia ${ }^{36}$. Durante el uso de glucocorticoides se debe considerar el tipo y el tiempo de acción para determinar los regímenes de tratamiento con insulina. Los glucocorticoides de acción intermedia como la metilprednisolona y prednisona alcanzan un pico en aproximadamente 4 a 8 horas, recomendándose insulina de acción intermedia (NPH); para los glucocorticoides de acción prolongada como la dexametasona o multidosis son preferibles los análogos de insulina de larga duración (glargina) ${ }^{37}$. Si el paciente es diabético y utilizaba terapia con insulina, esta deberá aumentarse al menos en un $20 \%$ durante el uso de esteroides.

La seguridad y eficacia de los tratamientos hipoglucemiantes no insulínicos en el ámbito hospitalario es un área de investigación activa ${ }^{38}$. Se ha reportado que el tratamiento con sitagliptina sola o en combinación con insulina basal es seguro y eficaz ${ }^{39}$. En pacientes no críticos con ingesta oral la utilización de metformina se ha asociado con efectos benéficos secundarios a su potencial inmunomodulación que genera reducción de citocinas proinflamatorias (como el factor de necrosis tumoral alfa y la interleucina 6$)^{40,41}$. Se ha sugerido que la utilidad de los inhibidores de la dipeptidil peptidasa-4 (DPP4) en pacientes diabéticos con COVID-19, acorde a modelos experimentales que sugieren a la DPP4 como correceptor para la entrada viral del SARS-CoV2 ${ }^{42}$, además de sus efectos glucorreguladores, antiinflamatorios y cardiometabólicos ${ }^{43-45}$. Sin embargo, se requieren más estudios para validar su efecto potencial ${ }^{46}$.

El control y monitorización de los tres casos se realizó con mediciones prepandiales, debido a que todos estaban comiendo y la administración de insulina era subcutánea, sin embargo, en pacientes que no estén comiendo debe de llevarse a cabo al menos cada 4 a 6 horas o en aquellos casos en que la terapia se lleve a cabo con insulina i.v. debe evaluarse cada 30 a 120 minutos. En caso de sospecharse o documentarse hipoglucemia deberá otorgarse tratamiento con base en la severidad; la vía oral es la recomendada en episodios leves a moderados, con consumo de hidratados de carbono simple de absorción rápida a dosis de 10 a 20 gramos. En los casos graves o aquellos que no respondan a la reposición de glucosa por vía oral puede administrarse $1 \mathrm{mg}$ de glucagón intramuscular o 25 a $50 \mathrm{~g}$ de solución glucosada al 50\%9,11,47.

Se recomienda realizar controles glucémicos durante el ingreso a todos los pacientes en nutrición enteral o parenteral o que utilicen otros medicamentos hiperglucemiantes (octreótido e inmunosupresores) durante las primeras 48 horas $^{9,11}$.

Se debe ajustar la dosis de insulina acorde a los resultados de glucemia: glucemia en ayuno y prepandial entre 100-140 mg/dL sin hipoglucemia en el día previo, mantener sin cambios; glucemia en ayuno y prepandial entre $140-180 \mathrm{mg} / \mathrm{dL}$ sin hipoglucemia en el día previo, incrementar dosis total de insulina (DTI) un 10\%; glucemia en ayuno y prepandial $>180 \mathrm{mg} / \mathrm{dL}$ sin hipoglucemia el día previo, incrementar la DTI un 20\%; glucemia en ayuno y prepandial entre $70-99 \mathrm{mg} / \mathrm{dL}$ sin hipoglucemia, disminuir la DTI en un 10\%; en presencia de hipoglucemia $(<70 \mathrm{mg} / \mathrm{dL}$ ), disminuir la DTI un $20 \%$. 
Antes del egreso del paciente que requirió hipoglucemiantes se evaluó una estrategia terapéutica multidisciplinaria enfocada en modificar el estilo de vida (hábitos dietéticos, actividad física y control de peso) y el tratamiento farmacológico más adecuado con la finalidad de controlar y evitar la progresión de la enfermedad, evitar los reingresos hospitalarios, el daño a órganos blanco y mejorar la calidad de vida. Se otorgó a todos los pacientes con hiperglucemia intrahospitalaria seguimiento ambulatorio ${ }^{48}$. Respecto a la elección de la terapia hipoglucemiante ambulatoria, se tomó en cuenta la edad del paciente, las comorbilidades asociadas, el riesgo cardiovascular y el nivel de $\mathrm{HbA} 1 \mathrm{c}^{20,35,49}$.

Debido a que nuestro caso era una paciente con diagnóstico nuevo de diabetes mellitus con $\mathrm{HbA} 1 \mathrm{c}>9 \%$, se decidió continuar con la dosis de insulina basalbolo; en caso de $\mathrm{HbA} 1 \mathrm{c}<9 \%$ se puede considerar hipoglucemiantes orales y en pacientes con diagnóstico previo de diabetes mellitus y niveles $\mathrm{HbA} 1 \mathrm{c}<7 \%$ reiniciar el régimen ambulatorio previo; si la HbA1c está entre el 7 y el $9 \%$, agregar el $50 \%$ de la dosis de insulina utilizada al tratamiento oral ambulatorio previo; y finalmente, si la $\mathrm{HbA1c}>9 \%$, continuar la dosis de insulina basal-bolo. En pacientes con diagnóstico nuevo de diabetes mellitus con niveles $\mathrm{HbA} 1 \mathrm{c}<9 \%$ se puede considerar hipoglucemiantes orales y con $\mathrm{HbA} 1 \mathrm{c}>9 \%$ continuar la dosis de insulina basal-bolo ${ }^{20,35}$.

\section{CONCLUSIONES}

Durante la actual pandemia de COVID-19 en nuestra unidad se ha observado un incremento en los casos de hiperglucemia, asociándose a una enfermedad más grave, con mayor estancia hospitalaria, incremento en el riesgo de ventilación mecánica y un peor pronóstico, estando la mayoría de las hiperglucemias relacionadas con diabetes mellitus con o sin diagnóstico previo y relacionadas con el uso de glucocorticoides o debido a una enfermedad grave. Por ello nos dimos a la tarea de documentar y resaltar la importancia de esta patología.

Los casos presentados sugieren que la hiperglucemia es una entidad frecuente en los pacientes con
COVID-19, con o sin diabetes y podría otorgar un peor pronóstico. Se deben considerar el efecto hiperglucémico de los glucocorticoides, la gravedad de la enfermedad, la disfunción pancreática por COVID-19 y la presencia de diabetes durante la monitorización y tratamiento de la hiperglucemia intrahospitalaria, recomendándose como parte del abordaje diagnóstico la realización de $\mathrm{HbA} 1 \mathrm{c}$ y ante la sospecha de disfunción pancreática solicitar amilasa y lipasa. La evidencia disponible es insuficiente para recomendar el uso de hipoglucemiantes orales en pacientes hospitalizados con COVID-19. La insulina es el agente de elección en el tratamiento de pacientes hospitalizados. A pesar de los grandes avances en el entendimiento de los procesos infecciosos agudos, es evidente que existen aún importantes áreas de oportunidad, por lo cual es recomendable realizar estudios que evalúen la asociación entre la presencia de hiperglucemia y la COVID-19.

\section{FINANCIAMIENTO}

Los autores no recibieron patrocinio para llevar a cabo este artículo

\section{CONFLICTO DE INTERESES}

Los autores declaran no tener conflicto de intereses.

\section{RESPONSABILIDADES ÉTICAS}

Protección de personas y animales. Los autores declaran que para esta investigación no se han realizado experimentos en seres humanos ni en animales.

Confidencialidad de los datos. Los autores declaran que han seguido los protocolos de su centro de trabajo sobre la publicación de datos de pacientes.

Derecho a la privacidad y consentimiento informado. Los autores han obtenido el consentimiento 
informado de los pacientes y/o sujetos referidos en el artículo. Este documento obra en poder del autor de correspondencia.

\section{BIBLIOGRAFÍA}

1. Zhou J, Tan J. Diabetes patients with COVID-19 need better blood glucose management in Wuhan, China. Metabolism. 2020;107:154216.

2. Bode B, Garrett V, Messler J, McFarland R, Crowe J, Booth R, et al. Glycemic characteristics and clinical outcomes of COVID-19 patients hospitalized in the United States. J Diabetes Sci Technol. 2020;14(4):813-21.

3. Bornstein SR, Rubino F, Khunti K, Mingrone G, Hopkins D, Birkenfeld AL, et al. Practical recommendations for the management of diabetes in patients with COVID-19. Lancet Diabetes Endocrinol. 2020;8(6):546-50.

4. Yang JK, Lin SS, Ji XJ, Guo LM. Binding of SARS coronavirus to its receptor damages islets and causes acute diabetes. Acta Diabetologica. 2010;47(3):193-9.

5. Pal R, Bhadada SK. COVID-19 and diabetes mellitus: An unholy interaction of two pandemics. Diabetes Metab Syndr. 2020:14(4):513-7.

6. Bommer C, Heesemann E, Sagalova V, Manne-Goehler J, Atun R, Bärnighausen $T$, et al. The global economic burden of diabetes in adults aged 20-79 years: a cost-of-illness study. Lancet Diabetes Endocrinol. 2017;5(6):423-30

7. Draznin B, Gilden J, Golden SH, Inzucchi SE. Pathways to quality inpatient management of hyperglycemia and diabetes: A call to action. Diabetes Care. 2013;36(7):1807-14.

8. Wu Z, McGoogan J. Characteristics of and important lessons from the coronavirus disease 2019 (COVID-19) outbreak in China: Summary of a Report of 72314 Cases From the Chinese Center for Disease Control and Prevention. JAMA. 2020;323(13):1239-42.

9. Umpierrez GE, Hellman R, Korytkowski MT, Kosiborod M, Maynard GA, Montori VM, et al. Management of hyperglycemia in hospitalized patients in non-critical care setting: An endocrine society clinical practice guideline. J Clin Endocrinol Metab. 2012;97(1):16-38.

10. Zhou K, Al-Jaghbeer MJ, Lansang MC. Hyperglycemia management in hospitalized patients with COVID-19. Cleve Clin J Med. 2020 Jul 17. doi: 10.3949/ccjm.87a.ccc012. Online ahead of print.

11. American Diabetes Association. Classification I. Standards of medical care in diabetes-2014. Diabetes Care. 2014;37(1):14-80.

12. Sardu C, D'Onofrio N, Balestrieri ML, Barbieri M, Rizzo MR, Messina V, et al. Outcomes in patients with hyperglycemia affected by COVID-19: Can we do more on glycemic control? Diabetes Care. 2020;43(7):1408-15.

13. Bello-Chavolla OY, Bahena-López JP, Antonio-Villa NE, Vargas-Vázquez A, González-Díaz A, Márquez-Salinas A, et al. Predicting mortality due to SARS-CoV-2: A mechanistic score relating obesity and diabetes to COVID-19outcomes in Mexico. JClin Endocrinol Metab. 2020;105(8):dgaa346.

14. Guo W, Li M, Dong Y, Zhou H, Zhang Z, Tian C, et al. Diabetes is a risk factor for the progression and prognosis of COVID-19. Diabetes Metab Res Rev. 2020 Mar 31:e3319. doi: 10.1002/dmrr.3319. Online ahead of print.

15. Mader JK, Brix J, Aberer F, Vonbank A, Resl M, Pieber TR, et al. Hospital diabetes management (Update 2019). Wien Klin Wochenschr. 2019;131(1):200-11.

16. Álvarez-Rodríguez E. Manejo de la hiperglucemia en la urgencia hospitalaria. Revista Diabetes Práctica. 2017;08(03):97-144.

17. Saigí I, Pérez A. Hiperglucemia inducida por glucocorticoides. Rev Clin Esp. 2010;210(8):397-403.

18. van Raalte $\mathrm{DH}$, Diamant M. Steroid diabetes: From mechanism to treatment? Neth J Med. 2014;72(2):62-72.

19. Tina Kurir, Josko Bozić, Anita Markotić AN. New insights in steroid diabetes. Acta Med Croatica. 2012;66(4):303-10.

20. Pasquel F, Umpierrez GE. Manejo de la hiperglucemia en el paciente hospitalizado. Medicina (Buenos Aires). 2010;70(3):275-83.

21. Roca H, Riera M, Palau V, Pascual J, Soler M. Characterization of ACE and ACE2 expression within different organs of the NOD mouse. Int J Mol Sci. 2017;18(3):563.

22. Cole S, Laviada-Molina H, Serres-Perales J, Rodriguez-Ayala E, Bastarrachea R. The COVID-19 pandemic during the time of the diabetes pandemic: Likely fraternal twins? Pathogens. 2020;9(5):1-13.

23. Rubino F, Amiel S, Zimmet P, Alberti G, Bornstein S, Eckel RH, et al. Newonset diabetes in Covid-19. N Engl J Med. 2020;383(8):789-90.
24. O'Sullivan E, Duignan J, O'Shea P, Griffin D, Dinneen SF. Evaluating hyperglycaemia in the hospitalised patient: Towards an improved system for classification and treatment. Ir J Med Sci. 2014;183(1):65-9.

25. Manley SE, O'Brien KT, Quinlan D, Round RA, Nightingale PG, Ali F, et al. $\mathrm{Can} \mathrm{HbA} 1 \mathrm{c}$ detect undiagnosed diabetes in acute medical hospital admissions? Diabetes Res Clin Pract. 2016;115:106-14.

26. De'Marziani G, Elbert A. Hemoglobina glicada. Utilidad y limitaciones en paciente con enfermedad renal crónica. Rev Nefrol Dial Traspl. 2018;38(1):65-83.

27. Wang $F$, Wang $H$, Fan J, Zhang $Y$, Wang $H$, Zhao $Q$. Pancreatic injury patterns in patients with coronavirus disease 19 pneumonia. Gastroenterology. 2020;159(1):367-70.

28. Mazrouei S, Saeed G, Al Helali A. COVID-19-associated acute pancreatitis: a rare cause of acute abdomen. Radiol Case Rep. 2020;15(9):1601-3.

29. American Diabetes Association. Diabetes care in the hospital: Standards of Medical Care in Diabetes-2018. Diabetes Care. 2018;41(1):S144-51.

30. American Diabetes Association. Diabetes Care in the Hospital: Standards of Medical Care in Diabetes-2020. Diabetes Care. 2020;43:S193-202.

31. Moghissi ES. Addressing hyperglycemia from hospital admission to discharge. Current Curr Med Res Opin. 2010;26(3):589-98.

32. Malcolm J, Halperin I, Miller DB, Moore S, Nerenberg KA, Woo V, et al. In-hospital management of diabetes. Can J Diabetes. 2018;42:S115-23.

33. Umpierrez G, Smiley D, Hermayer K, Khan A, Olson DE, Newton C, et al. Randomized study comparing a basal-boluswith a basal plus correction insulin regimen for the hospital management of medical and surgical patients with type 2 diabetes: Basal plus trial. Diabetes Care. 2013; 36(8):2169-74.

34. Umpierrez G, Smiley D, Jacobs S, Peng L, Temponi A, Mulligan P, et al. Randomized study of basal-bolus insulin therapy in the inpatient management of patients with type 2 diabetes undergoing general surgery (RABBIT 2 surgery). Diabetes Care. 2011;34(2):256-61.

35. Román A, Cardona A, Gutiérrez J, Palacio A. Management of diabetes in hospitals. Rev Fac Med. 2018;66(3):385-92.

36. Rafique S, Ahmed F. A case of combined diabetic ketoacidosis and hyperosmolar hyperglycemic state in a patient with COVID-19. Cureus. 2020;19(7):7-11.

37. Kwon S, Hermayer KL. Glucocorticoid-induced hyperglycemia. Am J Med Sci. 2013;345(4):274-7.

38. Pasquel F, Fayfman M, Umpierrez GE. Debate on insulin vs non-insulin use in the hospital setting - Is it time to revise the guidelines for the management of inpatient diabetes? Curr Diab Rep. 2019;19(9):65.

39. Umpierrez G, Gianchandani R, Smiley D, Jacobs S, Wesorick DH, Newton $C$, et al. Safety and efficacy of sitagliptin therapy for the inpatient management of generalmedicine and surgery patients with type 2 diabetes: A pilot, randomized, controlled study. Diabetes Care. 2013; 36(11):3430-5.

40. Kim J, Kwak HJ, Cha JY, Jeong YS, Rhee SD, Kim KR, et al. Metformin suppresses lipopolysaccharide (LPS)-induced inflammatory response in murine macrophages via Activating Transcription Factor-3 (ATF-3) induction. J Biol Chem. 2014;289(33):23246-55.

41. Kajiwara C, Kusaka Y, Kimura S, Yamaguchi T, Nanjo Y, Ishii Y, et al. Metformin mediates protection against Legionella Pneumonia through activation of AMPK and mitochondrial reactive oxygen species. J Immunol. 2018;200(2):623-31.

42. Vankadari N, Wilce JA. Emerging Wuhan (COVID-19) coronavirus: glycan shield and structure prediction of spike glycoprotein and its interaction with human CD26. Emerg Microbes Infect. 2020;9(1):601-4.

43. Drucker D. Coronavirus infections and type 2 diabetes-Shared pathways with therapeutic implications. Endocr Rev. 2020;41(3):457-0.

44. Bassendine M, Bridge S, McCaughan G, Gorrell M. COVID-19 and comorbidities: A role for dipeptidyl peptidase 4 (DPP4) in disease severity? J Diabetes. 2020;12(9):649-58.

45. Mulvihill EE, Drucker DJ. Pharmacology, physiology, and mechanisms of action of dipeptidyl peptidase-4 inhibitors. Endocr Rev. 2014;35(6):992-1019.

46. Filardi T, Morano S. COVID-19: is there a link between the course of infection and pharmacological agents in diabetes? J Endocrinol Invest. 2020;43(8):1053-60.

47. Nares M, González A, Martínez F, Morales M. Hipoglucemia: el tiempo es cerebro. ¿Qué estamos haciendo mal? Medicina Interna de México. 2018;34(6):881-95.

48. Guadalupe M, Martínez C, Gutiérrez SAG, Gabriela M, Craviotto L, Rosas $A$, et al. Manejo de la hiperglucemia en el paciente hospitalizado. Medicina Interna de México. 2012;28(2):124-53.

49. Hawkins K, Donihi AC, Korytkowski MT. Glycemic management in medical and surgical patients in the Non-ICU setting. Curr Diab Rep. 2013; 13(1):96-106 